SUPPORTING INFORMATION

Marine Phages as Tracers: Effects of Size, Morphology and Physico-Chemical Surface Properties on Transport in A Porous Medium

Nawras Ghanem ${ }^{1}$, Bärbel Kiesel ${ }^{1}$, René Kallies ${ }^{1}$, Hauke Harms ${ }^{1,2}$, Antonis Chatzinotas ${ }^{1,2}$ and Lukas Y. Wick ${ }^{1 *}$

${ }^{1}$ Helmholtz Centre for Environmental Research - UFZ, Department of Environmental Microbiology, Permoserstraße 15, 04318 Leipzig, Germany.

${ }^{2}$ German Centre for Integrative Biodiversity Research (iDiv) Halle-Jena-Leipzig, Deutscher Platz 5e, 04103 Leipzig, Germany

The Supporting Information has 9 pages, one table and three figures. It also contains additional information on theory and calculations on phage-collector interactions.

* Corresponding author: Helmholtz Centre for Environmental Research - UFZ. Department of Environmental Microbiology; Permoserstrasse 15; 04318 Leipzig, Germany. phone: +49 341235 1316, fax: +49 34123545 1316, e-mail: lukas.wick@ufz.de. 


\section{THEORY AND CALCULATIONS}

\section{Calculation of the collision efficiency $\left(\alpha_{t}\right)$}

The collision efficiency $\left(\alpha_{t}\right)$ of phages was calculated applying the colloid filtration theory. ${ }^{1}$ The collision efficiency $\alpha_{t}$ of phage is defined as the ratio of the experimental single-collector removal efficiency $\left(\eta_{t}\right)$ to the predicted single-collector contact efficiency $\left(\eta_{\text {trans }}\right)$. Therefore, $\alpha_{t}$ represents the relative affinity of phage for the packing material and $\alpha_{\mathrm{t}, 0}$ represents the initial collision efficiency. The collision efficiency was calculated from experimental phage breakthrough curves (eq. $\mathrm{S} 1)^{2}$

$\alpha_{\mathrm{t}}=-\frac{2}{3} \frac{\mathrm{a}_{\mathrm{s}}}{(1-\varepsilon) \mathrm{L} \eta_{\mathrm{trans}}} \ln \left(C / C_{0}\right)$

where $C$ is the effluent phage concentration, $C_{0}$ the influent phage concentration, $\varepsilon$ the porosity of the packed bed, $a_{s}$ the radius of the sand particles, $L$ the length of the column, and $\eta_{\text {trans }}$ the predicted single-collector contact efficiency. Values of $\eta_{\text {trans }}$ were calculated as a sum of each transport mechanism taking into account the contributions of convection, diffusion, van der Waals attraction, and sedimentation. ${ }^{2}$ For the calculations, we assumed spheres of identical size sand particle (mean diameter $0.29 \mathrm{~mm}$ ), and identical radius of the phage. $\eta_{\text {trans }}$ was approximated by applying the following equation (eq. S2):

$\eta_{\text {trans }}=2.4 A_{s}^{1 / 3} N_{R}^{-0.081} N_{P e}^{-0.715} N_{v d W}^{0.052}+0.55 A_{S} N_{R}^{1.675} N_{A}^{0.125}+0.22 N_{R}^{-0.24} N_{G}^{1.11} N_{v d W}^{0.053}$

Where $N_{R}=\frac{a_{p}}{a_{S}}$ is the aspect ratio, $N_{P e}=\frac{U a_{S}}{D_{\infty}}$ is the Peclet number, $N_{v d W}=\frac{A_{123}}{K T}$ is the van der Waals number, $N_{g r}=\frac{4}{3} \frac{\pi a_{p}^{4}\left(\rho_{p}-\rho_{f}\right)}{K T}$ is the gravitational number and $A_{\mathrm{S}}$ is the a porosity-dependent parameter which calculated applying equation (eq. S3)

$A_{s}=\frac{2\left(1-\varepsilon^{5}\right)}{2-3+3 \varepsilon^{5}-2 \varepsilon^{6}}$

The latest dimensionless parameters were calculated as described by Tufenkji \& Elimelech $2004^{2}$ with $a_{\mathrm{p}}$ and $a_{\mathrm{s}}$ being the radii of phages and the sand particles $\left(a_{s}=1.4 \times 10^{-4} \mathrm{~m}\right)$, respectively, $U$ the fluid approach velocity $\left(0.7 \times 10^{-2} \mathrm{~cm} \mathrm{~s}^{-1}\right), D_{\infty}$ the bulk diffusion coefficient, ${ }^{1} k$ is the Boltzmann constant $\left(1.38 \times 10^{-23} \mathrm{~J} \mathrm{~K}^{-1}\right), \mathrm{T}$ is the fluid absolute temperature 293K, $\rho_{\mathrm{p}}$ the density of phage particles (we used the density of MS2 phage for all phages; $\rho_{\mathrm{p}}$ $\left.=1420 \mathrm{~kg} \mathrm{~m}^{-3}\right){ }^{3} \rho_{\mathrm{f}}$ the density of the fluid, and $\mathrm{A}_{132}$ the Hamaker constant as described by eq. $\mathrm{S} 4:^{4}$ 
$A_{123}=\left(\sqrt{A_{11}}-\sqrt{A_{33}}\right)\left(\sqrt{A_{22}}-\sqrt{A_{33}}\right)$

Here, $A_{i i}$ denotes the individual Hamaker constant of phages $\left(A_{11}\right)$, sand $\left(A_{22},\right)$ and water $\left(A_{33}\right)$, respectively. $A_{33}$ was taken from literature ${ }^{4}$ whereas $A_{11}$ and $A_{22}$ were obtained by eq. S5.

$$
A_{i i}=6 \pi l_{0}^{2} \gamma_{i}^{L W}
$$

According to Fowkes, ${ }^{5}$ the value of $6 \pi l_{0}^{2}$ equals $1.44 \times 10^{-18} \mathrm{~m}^{2}$, with $l_{0}$ being the equilibrium separation distances between the phage and the sand particle $(0.157 \mathrm{~nm}){ }^{6}$

\section{Calculation of XDLVO interaction energy of Phage adhesion}

The XDLVO interaction energy, $G_{\mathrm{XDLVO}}$ is the sum of the electrostatic repulsion $\left(G_{\mathrm{EDL}}\right)$, the Lifshitzvan der Waals $\left(G_{\mathrm{LW}}\right)$ and the acid-base $\left(G_{\mathrm{AB}}\right)$ interaction energy. The $G_{\mathrm{EDL}}$ and the $G_{\mathrm{LW}}$ depend on the separation distance $\mathrm{h}[\mathrm{nm}]$ between two surfaces ${ }^{7,8}$ whereas $G_{\mathrm{AB}}$ compares the energy status between attached and nonattached situations (eq. S6):

$$
\mathrm{G}_{\mathrm{XDLVO}}(h)=\mathrm{G}_{\mathrm{AB}}+\mathrm{G}_{\mathrm{EDL}}(h)+\mathrm{G}_{\mathrm{LW}}(h)
$$

Acid-base interaction energy $\left(G_{\mathrm{AB}}\right)$. The acid-base interaction energy $\left(G_{\mathrm{AB}}\right)$ was calculated by applying the (eq. S7) ${ }^{9,10}$

$G_{A B} \pi=2 \pi a_{P} \Delta G^{A B} \lambda \exp \left(\frac{l_{0}-h}{\lambda}\right)$

Where $a_{P}$ is the radius of phages, and $h$ is the separation distance between the phage and the surface. The $\lambda$ is the characteristic decay length of $A B$ interaction in water (estimated to be $0.6 \mathrm{~nm})^{11}$. The acid-base interaction energy depends on the Gibbs free energy of the phage and the sand as given by (eq. S7). $\Delta G^{\mathrm{AB}}$ is the acid-base component of the free energy interaction at contact given by eq. $\mathrm{S} 8:^{11,12}$

$$
\begin{aligned}
& \Delta G^{A B}=\left[2\left(\sqrt{\gamma_{P}^{+}}-\sqrt{\gamma_{s}^{+}}\right)\left(\sqrt{\gamma_{P}^{-}}-\sqrt{\gamma_{s}^{-}}\right)-\left(\sqrt{\gamma_{P}^{+}}-\sqrt{\gamma_{\mathrm{l}}^{+}}\right)\left(\sqrt{\gamma_{P}^{-}}-\sqrt{\gamma_{\mathrm{l}}^{-}}\right)-\left(\sqrt{\gamma_{s}^{+}}-\right.\right. \\
& \left.\left.\sqrt{\gamma_{\mathrm{l}}^{+}}\right)\left(\sqrt{\gamma_{s}^{-}}-\sqrt{\gamma_{\mathrm{l}}^{-}}\right)\right]
\end{aligned}
$$

The measured contact angles $(\theta)$ of phages, membrane filters and sand surfaces using water, formamide and methylene iodide as liquids were used to calculate the surface Gibbs free 
energies of phages $\gamma_{P}$ and the sand surface $\gamma_{s}\left(\mathrm{~mJ} \mathrm{~m}^{-2}\right)$ by applying the Young equation according to eq. S9:

$\cos (\theta)=-1+2 \frac{\sqrt{\gamma_{P}^{L W} \gamma_{l}^{L W}}}{\gamma_{l}^{\text {total }}}+2 \frac{\sqrt{\gamma_{P}^{+} \gamma_{\mathrm{l}}^{-}}}{\gamma_{\mathrm{l}}^{\text {total }}}+2 \frac{\sqrt{\gamma_{P}^{-} \gamma_{l}^{+}}}{\gamma_{\mathrm{l}}^{\text {total }}}$

Equation S10 represents the total surface Gibbs free energiy $\left(\gamma^{\text {total }}\right)$ which is separated in a Lifshitz-van der Waals $\left(\gamma^{\mathrm{LW}}\right)$ and an acid-base component $\left(\gamma^{\mathrm{AB}}\right)$. The electron acceptor and the electron donor components of acid-base surface energy $\gamma^{+}$and $\gamma^{-}$is shown in eq. S11.

$\gamma^{\text {total }}=\gamma^{A B}+\gamma^{L W}$

$\gamma_{i}^{A B}=2 \sqrt{\gamma_{i}^{+} \gamma_{i}^{-}}$

The parameters $\gamma_{\mathrm{p}}, \gamma_{\mathrm{p}}^{\mathrm{LW}}, \gamma_{\mathrm{p}}^{+}, \gamma_{\mathrm{p}}^{-}$of phages were calculated following van Oss et $\mathrm{al}^{6}$, while for water, formamide and methyleneiodide we used the literature data ${ }^{13}$.

Electrostatic repulsion energy, $\left(\mathrm{G}_{\mathrm{EDL}}\right)$. The electrostatic repulsion energy between phages and the sand surface was calculated by eq. $\mathrm{S} 12:^{7}$

$G_{E D L}=\pi \varepsilon_{0} \varepsilon_{r} a_{p}\left\{2 \zeta_{p} \zeta_{s} \ln \left[\frac{1+\exp (-\kappa h)}{1-\exp (-\kappa h)}\right]+\left(\zeta_{p}^{2}+\zeta_{s}^{2}\right) \ln [1-\exp (-2 \kappa h)]\right.$

where $\kappa^{-1}$ is the thickness of the electrical double layer (EDL, nm) as calculated by the GuoyChapman theory with $C$ and $\mathrm{z}$ being the molar bulk concentration and the charge number of the electrolytes, respectively (eq. S13).

$\kappa^{-1}=\left[3.29 z C^{1 / 2}\right]^{-1}$

For a $100 \mathrm{mM}$ buffer solution a $\kappa^{-1}$ of $0.65 \mathrm{~nm}$ was calculated. ${ }^{13}$

Lifshitz-van der Waals interaction energy $\left(G_{L W}\right)$. Using the values of the effective Hamaker constant, the Lifshitz-van der Waals interaction energy can be approximated by eq. S14: ${ }^{12,13}$

$G_{L W}=-\frac{A_{132}}{6}\left[\frac{2 a_{p}\left(h+a_{p}\right)}{h\left(h+a_{p}\right)}-\ln \left(\frac{h+2 a_{p}}{h}\right)\right]$ 


\section{TABLES AND FigureS}

Table S1. Overview of the surface Gibbs free energy $(\gamma)$ and the contact angles of water $\theta_{\mathrm{w}}$, methylene iodide $\theta_{\mathrm{m}}$ and formamide $\theta_{\mathrm{f}}$ of sand and lawns of studied phages.

\begin{tabular}{lllllllll}
\hline Phage name & \multicolumn{7}{c}{ Contact angle $(\Theta)$} & \multicolumn{6}{c}{ Surface free energy $\left(\mathrm{mJ} \mathrm{m}^{-2}\right)^{*}$} \\
& $\Theta_{\mathrm{w}}$ & $\Theta_{\mathrm{f}}$ & $\Theta_{\mathrm{m}}$ & $r^{-}$ & $r^{+}$ & $r^{\mathrm{AB}}$ & $r^{\mathrm{w} \mathrm{w}}$ & $r^{\text {Tot }}$ \\
\hline Water & & & & 25.5 & 25.50 & 51.0 & 21.8 & 72.8 \\
Formamide & & & & 39.6 & 2.3 & 19.0 & 39.0 & 58.0 \\
Methylene iodide & & & & $<0.1$ & $<0.1$ & $\approx 0$ & 50.8 & 50.8 \\
Membranfilter Anodisc 25 & 23 & & & & & & & \\
PSA-HM1 & 40 & 33 & 48 & 36.4 & 1.2 & 13.2 & 35.6 & 48.8 \\
PSA-HP1 & 39 & 42 & 50 & 46.5 & 0.3 & 7.4 & 34.5 & 41.9 \\
PSA-HS2 & 40 & 31 & 43 & 36.2 & 0.96 & 11.5 & 38.0 & 49.5 \\
VB_PSPS-H40/1 & 53 & 36 & 49 & 22.6 & 1.8 & 6.8 & 34.8 & 41.6 \\
vB_PSPS-H6/1 & 58 & 38 & 47 & 18 & 1.62 & 15.3 & 35.7 & 51.0 \\
H3/49 & 61 & 55 & 51 & 25.0 & 0.06 & 2.4 & 33.9 & 36.3 \\
T4 & 95 & 61 & 40 & 0.1 & 0.30 & 0.2 & 39.5 & 39.7 \\
MS2 & 84 & 63 & 45 & 5.2 & 0.00 & 0.7 & 37.1 & 37.8 \\
Sand & 71 & 60 & 71 & 15.4 & 1.60 & 9.9 & 22.7 & 32.6 \\
\hline
\end{tabular}

${ }^{*}$ Surface free energy data for water, formamide, methyleneiodide and glass taken from Sharma and Hanumantha Rao, $2003^{13}$. 

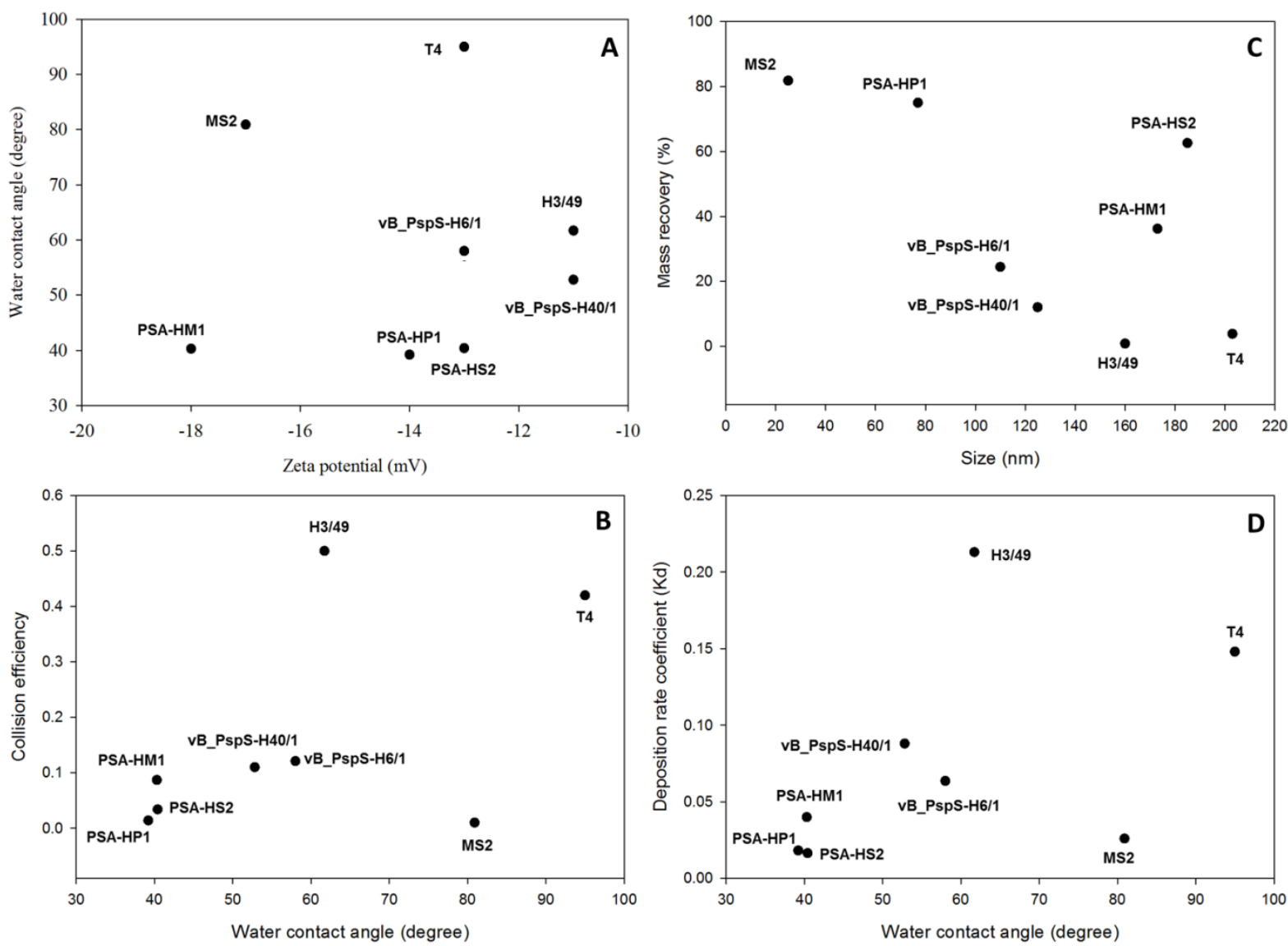

Figure S1. Panel A: Overview of the physico-chemical surface properties (water contact angle and zeta potential) of six marine and two nonmarine phages belonging to four different virus families: Myoviridae (H3/49, PSA-HM1, T4), Siphoviridae (vB_PspS-H40/1, vB_PspS-H6/1, PSA-HS2), Podoviridae (PSA-HP1) and Leviviridae (MS2). Panels B \& D correlate water contact angles of the phages with their collision efficiency, respectively, whereas panel $\mathrm{C}$ links the size of the phages with their mass recovery during transport experiments in sand-filled percolation columns. 

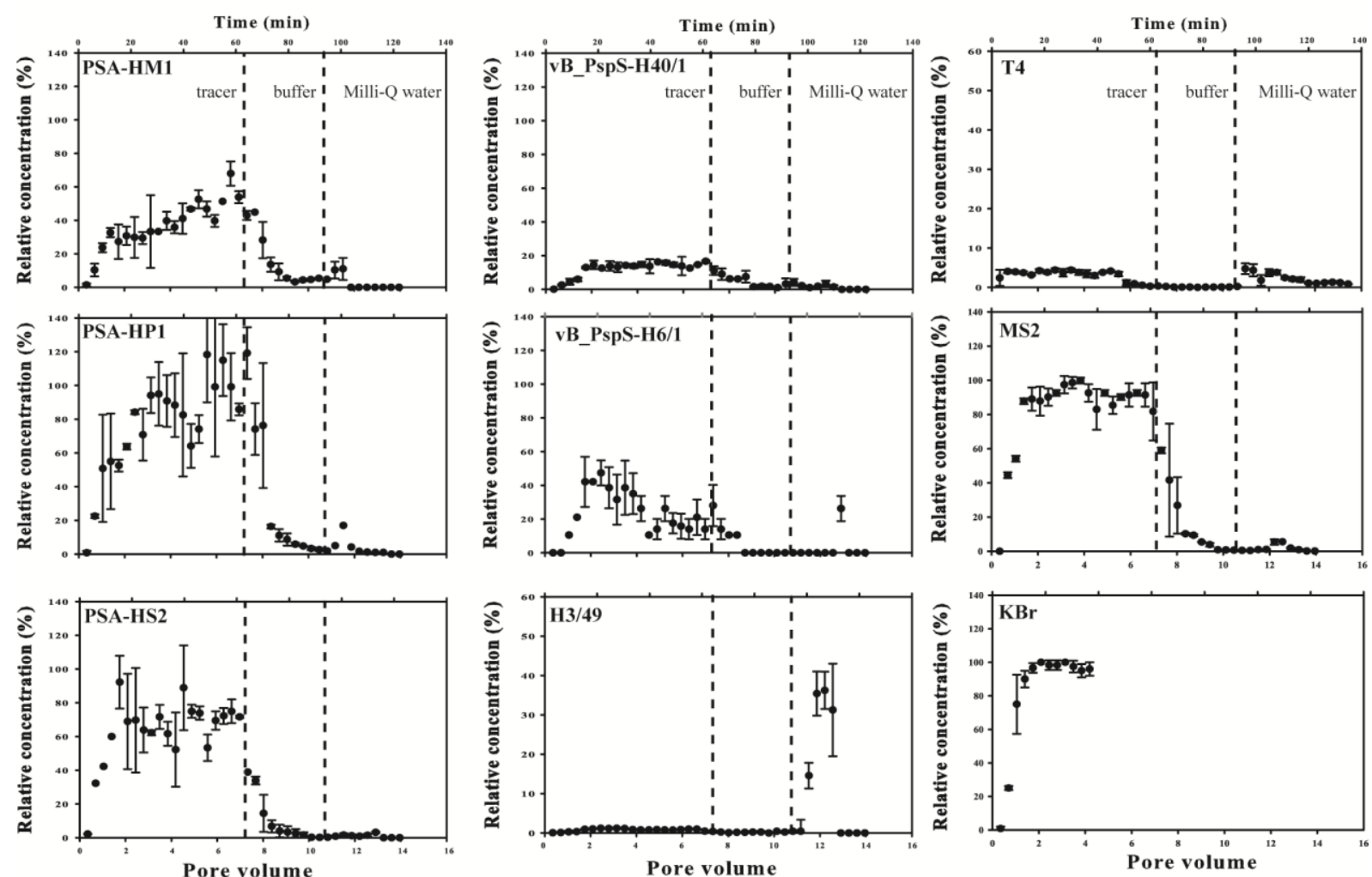

Figure S2. Plots of bromide breakthrough curve (right down) and of relative phage densities in the columns' outflow ( $\mathrm{C} / \mathrm{C} 0)$ against time (as normalized to the number of PV exchanged). The three different phases (loading with phage tracer; flushing with phage-free buffer, and flushing with Milli-Q water) of phage's breakthrough curves are marked by black vertical lines. Please note that phages T4 and H3/49 breakthrough curves have a different scale of their $y$-axis. 


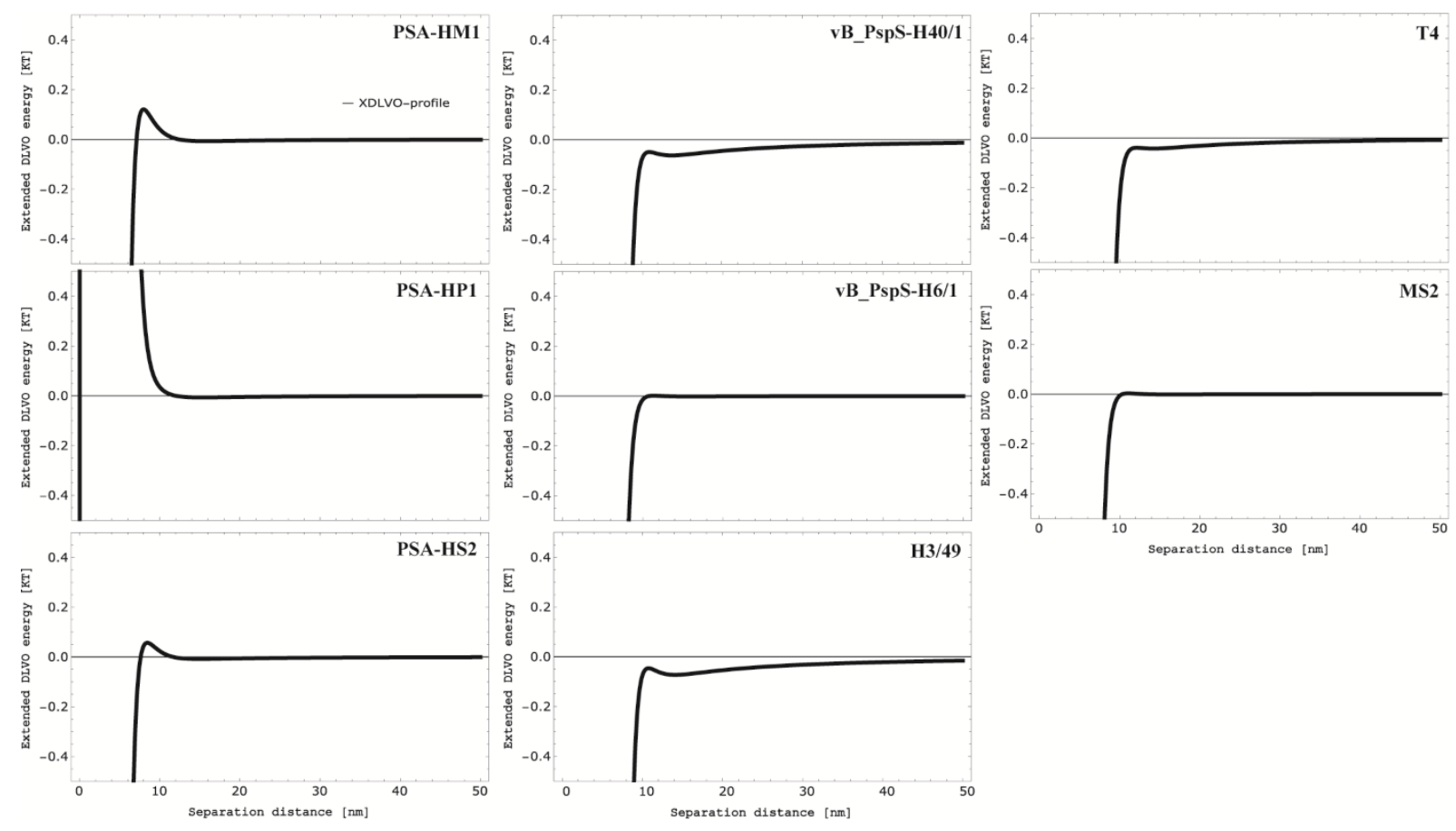

Figure S3. The XDLVO interaction energy profiles between sand particles and different phages. The interaction energy profiles show the overall interaction energy (solid line) as a function of distance particle $h(\mathrm{~nm})$ between the phage and the sand surface. 


\section{REFERENCES}

(1) Syngouna, V. I.; Chrysikopoulos, C. V. Transport of biocolloids in water saturated columns packed with sand: Effect of grain size and pore water velocity. J. Contam. Hydrol. 2011, 126 (3-4), 301-314.

(2) Tufenkji, N.; Elimelech, M. Correlation Equation for Predicting Single-Collector Efficiency in Physicochemical Filtration in Saturated Porous Media. Environ. Sci. Technol. 2004, 38 (2), 529-536.

(3) Walshe, G. E.; Pang, L.; Flury, M.; Close, M. E.; Flintoft, M. Effects of pH, ionic strength, dissolved organic matter, and flow rate on the co-transport of MS2 bacteriophages with kaolinite in gravel aquifer media. Water Res. 2010, 44 (4), 12551269.

(4) Van Oss, C. J.; Good, R. J.; Chaudhury, M. K. The role of van der Waals forces and hydrogen bonds in "hydrophobic interactions" between biopolymers and low energy surfaces. J. Colloid Interface Sci. 1986, 111 (2), 378-390.

(5) Fowkes, F. M. Attractive forces at interfaces. Ind. Eng. Chem. 1964, 56 (12), 40-52.

(6) Van Oss, C. J.; Chaudhury, M. K.; Good, R. J. Interfacial Lifshitz-van der Waals and polar interactions in macroscopic systems. Chem. Rev. 1988, 88 (6), 927-941.

(7) Boks, N. P.; Norde, W.; van der Mei, H. C.; Busscher, H. J. Forces involved in bacterial adhesion to hydrophilic and hydrophobic surfaces. Microbiology 2008, 154 (10), 31223133.

(8) Bergendahl, J.; Grasso, D. Prediction of colloid detachment in a model porous media: Thermodynamics. AIChE J. 1999, 45 (3), 475-484.

(9) Tadros, T. Interfacial forces in aqueous media, Carel J. van Oss, Marcel Dekker Inc., New York, 1994. J. Chem. Technol. Biotechnol. 1995, 64 (3), 311-311.

(10) Chrysikopoulos, C. V.; Syngouna, V. I. Attachment of bacteriophages MS2 and $\Phi$ X174 onto kaolinite and montmorillonite: Extended-DLVO interactions. Colloids Surf. B Biointerfaces 2012, 92, 74-83.

(11) van Oss, C. J.; Docoslis, A.; Wu, W.; Giese, R. F. Influence of macroscopic and microscopic interactions on kinetic rate constants: I. Role of the extended DLVO theory in determining the kinetic adsorption constant of proteins in aqueous media, using von Smoluchowski's approach. Colloids Surf. B Biointerfaces 1999, 14 (1-4), 99-104.

(12) Van Oss, C. J.; Giese, R. F.; Costanzo, P. M. DLVO and non-DLVO interactions in hectorite. Clays Clay Min. 1990, 38 (2), 151-159.

(13) Sharma, P. K.; Hanumantha Rao, K. Adhesion of Paenibacillus polymyxa on chalcopyrite and pyrite: surface thermodynamics and extended DLVO theory. Colloids Surf. B Biointerfaces 2003, 29 (1), 21-38. 